\title{
A Three-Dimensional Chaotic System and Its New Proposed Electronic Circuit
}

\author{
Maysoon M. Aziz, Dalya M. Merie \\ Department of Mathematics, College of Computer Sciences and Mathematics, University of Mosul, Mosul, Iraq \\ Email: aziz_maysoon@yahoo.com
}

How to cite this paper: Aziz, M.M. and Merie, D.M. (2020) A Three-Dimensional Chaotic System and Its New Proposed Electronic Circuit. Open Access Library Journal, 7: e6555.

https://doi.org/10.4236/oalib.1106555

Received: June 24, 2020

Accepted: July 26, 2020

Published: July 29, 2020

Copyright $\odot 2020$ by author(s) and Open Access Library Inc.

This work is licensed under the Creative Commons Attribution International License (CC BY 4.0).

http://creativecommons.org/licenses/by/4.0/

\section{(c) (i) Open Access}

\begin{abstract}
In this paper, new electronic circuit was designed with two-equilibrium as an engineering application on a three-dimensional chaotic system. The circuit consists of resistors, capacitors, voltages and operational amplifiers TL032CN. The adopted continuous-time chaotic dynamical system is with quadratic cross-product nonlinear terms and parameters. The basic characteristics of the proposed circuit model were analyzed in detail by equilibrium points, stability analysis, Lyapunov exponents and Kaplan-Yorke dimension. The results were simulated theoretically using MultiSIM 10, and it was well consistent with the results obtained from the Matlab program.
\end{abstract}

\section{Subject Areas}

Dynamical System

\section{Keywords}

Hyperchaotic System, Chaotic Attractors, Kaplan-Yorke Dimension, Circuit Simulation

\section{Introduction}

Chaos theory describes nonlinear dynamical systems that are very sensitive to initial conditions. Since the experimental discovery of a chaotic system by Lorenz, chaos theory has found applications in several areas in science and engineering [1]. Chaos is a phenomenon caused by the sensitivity of perturbation structural parameters and the initial conditions of some categories of dynamic systems [2] [3].

In last decades, chaotic circuit has received considerable interest in the researches due to the fact that they have been applied in abundant areas like in secure communications, simulating economical models, design of electronic cir- 
cuits, robotics, image processing, and neural networks [4] [5]. For this reason, a large number of chaotic systems, which are implemented in circuit, are reported in the literature [6].

Electrical laws are necessary to analyze any electrical circuit effectively and efficiently by determining different circuit parameters such as current, voltage power and resistance. These laws include Ohms law, Kirchhoff's current and voltage laws etc. [7].

This paper contains: Section 2, we description 3-D chaotic system; it is mainly consisted of six simple terms including two nonlinear terms. Section 3, an electronic circuit is designed to implement chaotic system (1). Section 4, we simulated the designed circuit by electronic simulation MultiSIM 10 program. And Section 5, we presented the conclusions.

\section{Description of Chaotic System with Two Equilibrium Points}

An autonomous 3-D dynamical system [8] with two quadratic cross-product nonlinear terms

$$
\begin{aligned}
& \dot{x}_{1}=\rho\left(x_{2}-x_{1}\right) \\
& \dot{x}_{2}=a x_{1}-\delta x_{1} x_{3} \\
& \dot{x}_{3}=\varphi x_{1} x_{2}-x_{3}
\end{aligned}
$$

where $x_{1} x_{3}, x_{1} x_{2}$ are the quadratic cross-product nonlinear terms in the dynamical system. The system (1) is chaotic when the parameters values $(\rho, a, \delta$ and $\varphi$ ) are taken as

$$
\rho=10, \delta=40, a=296.5, \varphi=10
$$

So for the given values of parameters, the Lyapunov exponents of system (1) are determined as

$$
L_{1}=2.509426, L_{2}=0.132019 \text { and } L_{3}=-11.818787 \text {. }
$$

Also, Kaplan-Yorke dimension of system (1) is calculated as

$$
D_{K Y}=2+\frac{L_{1}+L_{2}}{\left|L_{3}\right|}=2.22349544
$$

which shows hyper-chaotisity of system (1).

Therefore, the hyperchaotic system (1) has a strange attractor. For graphical results, we used Matlab and take initial states $\left.x\right|_{x_{1}(0), x_{2}(0), x_{3}(0)}=[-2,7,12]$.

Figure 1 shows chaotic attractor for system (1) in $\boldsymbol{R}^{3}$.

Figures 2(a)-(c) show the system (1) exhibit chaotic attractors in (a): $\left(x_{1}, x_{2}\right)$ plane, (b): $\left(x_{2}, x_{3}\right)$ plane, $(\mathrm{c}):\left(x_{1}, x_{3}\right)$ plane.

Now, the wave-form $x_{1}(t), x_{2}(t), x_{3}(t)$ for the system (1) has a non-periodic shape, shown in Figures $3(a)-(c)$ which is one of the basic characteristic behaviors of chaotic dynamical system.

\section{The Proposed Circuit of 3-D Chaotic System (1)}

An electronic circuit is designed to implement chaotic system (1). The circuit 
consists of electronic elements: capacitors, multipliers, resistors and operational amplifiers TL032CN.

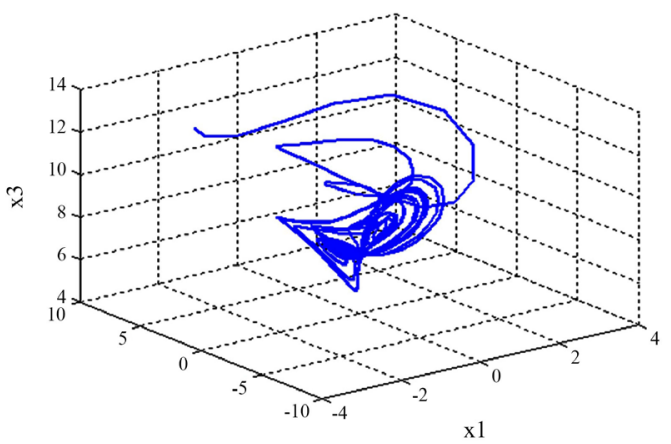

Figure 1. Chaotic attractor of system (1) with $(\rho, a, \delta$, $\phi)=(10,296.5,40,10)$ in $\left(X_{1}-X_{2}-X_{3}\right)$ space.

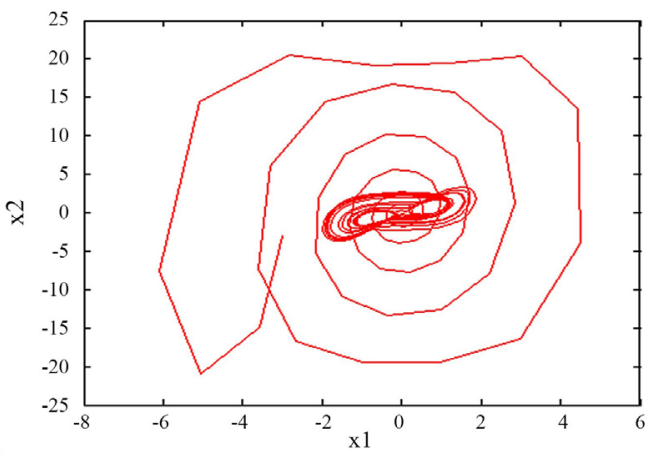

(a)

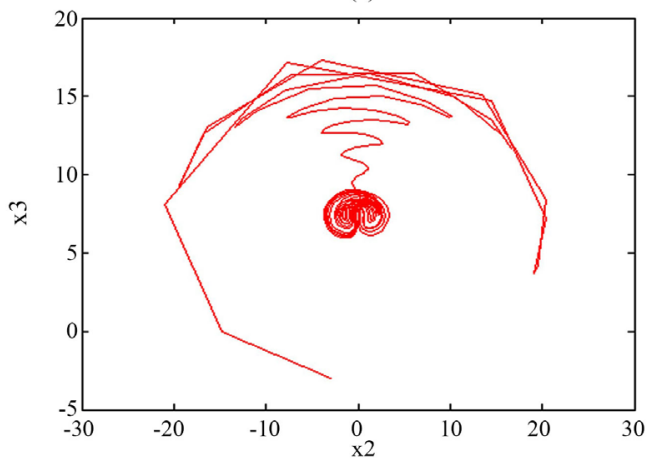

(b)

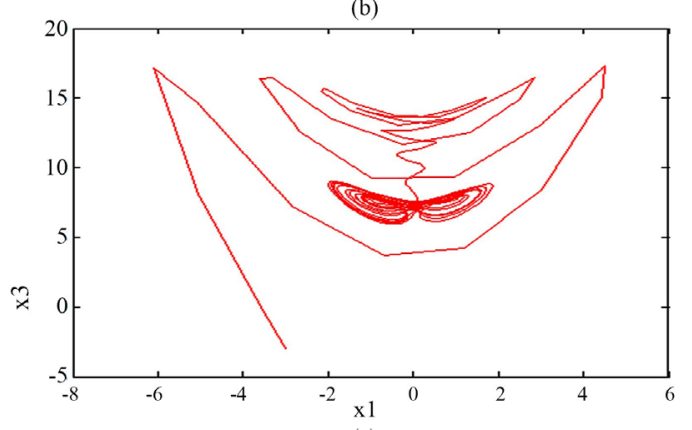

(c)

Figure 2. Chaotic attractor of system (1) with $(\rho, a, \delta$, $\phi)=(10,296.5,40,10)$ in (a) $\left(X_{1}-X_{2}\right)$ plane, (b) $\left(x_{2}-X_{3}\right)$ plane, (c) $\left(X_{1}-X_{3}\right)$ plane. 


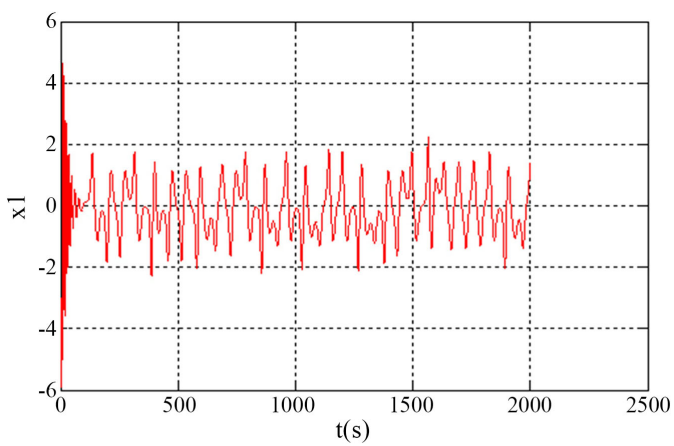

(a)

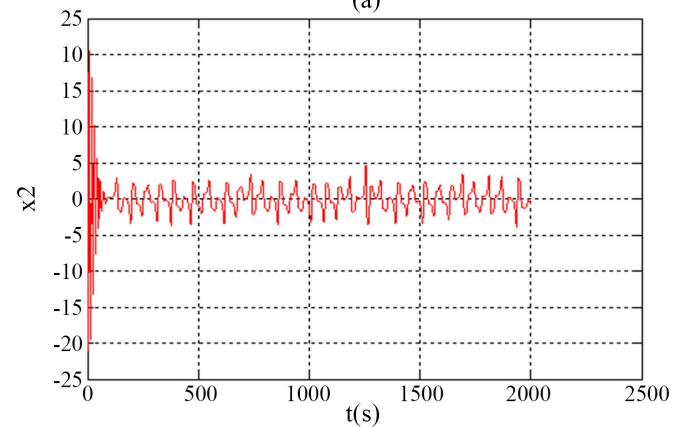

(b)

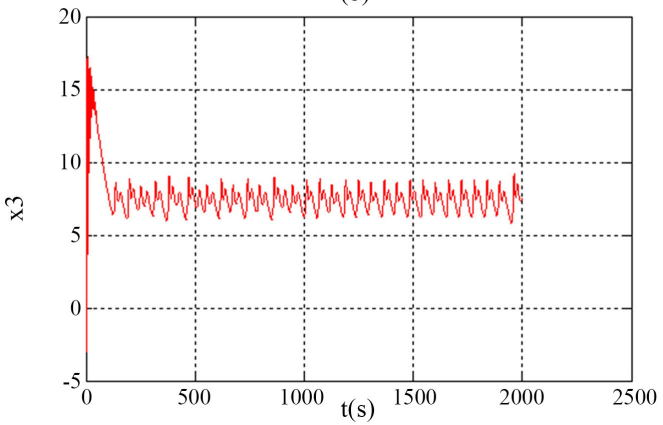

(c)

Figure 3. The wave-form of system (1). (a): Time versus $x_{1}$; (b): Time versus $x_{2}$; (c): Time versus $x_{3}$.

By applying Kirchhoff's laws [7], the corresponding circuit equations described as:

$$
\begin{aligned}
& \frac{\mathrm{d} V_{x_{1}}}{\mathrm{~d} t}=\frac{1}{R_{1} C_{1}}\left(V_{x_{2}}-V_{x_{1}}\right) \\
& \frac{\mathrm{d} V_{x_{2}}}{\mathrm{~d} t}=\frac{1}{R_{2} C_{2}} V_{x_{1}}-\frac{1}{R_{3} C_{2}} V_{x_{1}} V_{x_{3}} \\
& \frac{\mathrm{d} V_{x_{3}}}{\mathrm{~d} t}=\frac{1}{R_{4} C_{3}} V_{x_{1}} V_{x_{2}}-\frac{1}{R_{5} C_{3}} V_{x_{3}}
\end{aligned}
$$

where $V_{x_{1}}, V_{x_{2}}, V_{x_{3}}$ are the output voltages and $k_{m}=10 \mathrm{~V}$ is the fixed multipliers constant, hence the outputs are $V_{x_{1} x_{3}}=V_{x_{1}} V_{x_{3}} / k_{m}$ and $V_{x_{1} x_{2}}=V_{x_{1}} V_{x_{2}} / k_{m}$.

Voltages and time normalized by dimensionless states variables

$$
V_{x_{1}}=1 \mathrm{~V} \cdot x_{1}, V_{x_{2}}=1 \mathrm{~V} \cdot x_{2}, V_{x_{3}}=1 \mathrm{~V} \cdot x_{3}, t^{\prime}=\tau \cdot t=100 \mu \mathrm{s} \cdot t
$$

Substitute (4) in equations of system (3) we get: 


$$
\begin{aligned}
\frac{\mathrm{d} x_{1}}{\mathrm{~d} t^{\prime}} & =\frac{\tau}{R_{1} C_{1}}\left(x_{2}-x_{1}\right) \\
\frac{\mathrm{d} x_{2}}{\mathrm{~d} t^{\prime}} & =\frac{\tau}{R_{2} C_{2}} x_{1}-\frac{\tau}{R_{3} C_{2}} x_{1} x_{3} \\
\frac{\mathrm{d} x_{3}}{\mathrm{~d} t^{\prime}} & =\frac{\tau}{R_{4} C_{3}} x_{1} x_{2}-\frac{\tau}{R_{5} C_{3}} x_{3}
\end{aligned}
$$

Comparing system (1) with system (5) gives following conditions:

$$
\frac{\tau}{R_{1} C_{1}}=\rho, \frac{\tau}{R_{2} C_{2}}=a, \frac{\tau}{R_{3} C_{2}}=\delta, \frac{\tau}{R_{4} C_{3}}=\varphi, \frac{\tau}{R_{5} C_{3}}=1
$$

Take convenient values for capacitances and resistances as

$$
\begin{aligned}
& C_{1}=C_{2}=C_{3}=1 \mathrm{mF}, R_{1}=R_{4}=10 \Omega, R_{2}=337.268 \mathrm{~m} \Omega, \\
& R_{3}=2.5 \Omega, R_{5}=100 \Omega .
\end{aligned}
$$

We obtained the experimental electronic circuit (5) for system (1) with parameters $\rho=10, a=296.5, \delta=40, \varphi=10$.

\subsection{Equilibrium Points}

To find equilibrium points we need to solve the nonlinear equations as follows:

$$
\begin{aligned}
& \frac{\tau}{R_{1} C_{1}}\left(x_{2}-x_{1}\right)=0 \\
& \frac{\tau}{R_{2} C_{2}} x_{1}-\frac{\tau}{R_{3} C_{2}} x_{1} x_{3}=0 \\
& \frac{\tau}{R_{4} C_{3}} x_{1} x_{2}-\frac{\tau}{R_{5} C_{3}} x_{3}=0
\end{aligned}
$$

We get two equilibrium points

$$
E_{1}=\left(\frac{\sqrt{\frac{593}{2}}}{20}, \frac{\sqrt{\frac{593}{2}}}{20}, \frac{593}{80}\right), \quad E_{2}=\left(-\frac{\sqrt{\frac{593}{2}}}{20},-\frac{\sqrt{\frac{593}{2}}}{20}, \frac{593}{80}\right)
$$

\subsection{Stability Analysis}

\subsubsection{Characteristic Equation Roots}

The Jacobian matrix of system (5) is:

$$
\begin{gathered}
J=\left[\begin{array}{ccc}
-\frac{\tau}{R_{1} C_{1}} & \frac{\tau}{R_{1} C_{1}} & 0 \\
\frac{\tau}{R_{2} C_{2}}-\frac{\tau}{R_{3} C_{2}} x_{3} & 0 & -\frac{\tau}{R_{3} C_{2}} x_{1} \\
\frac{\tau}{R_{4} C_{3}} x_{2} & \frac{\tau}{R_{4} C_{3}} x_{1} & -\frac{\tau}{R_{5} C_{3}}
\end{array}\right] \\
J_{E_{1}}=\left[\begin{array}{ccc}
-10-\lambda & 10 & 0 \\
266.85 & -\lambda & -34.43835072 \\
8.609587679 & 8.609587679 & -1-\lambda
\end{array}\right]
\end{gathered}
$$


And

$$
J_{E_{2}}=\left[\begin{array}{ccc}
-10-\lambda & 10 & 0 \\
266.85 & -\lambda & 34.43835072 \\
-8.609587679 & -8.609587679 & -1-\lambda
\end{array}\right]
$$

Now, find characteristic equation by setting $\operatorname{det}(J-\lambda I)=0$, we get the same equation at $E_{1}$ and $E_{2}$ :

$$
\lambda^{3}+11 \lambda^{2}-2362 \lambda+3261.5=0
$$

We obtain the same eigenvalues at equilibrium points $E_{1}$ and $E_{2}$ :

$$
\lambda_{1}=1.39097, \lambda_{2}=42.622, \lambda_{3}=-55.013
$$

Since, there are positive eigenvalues, so system (5) is unstable.

\subsubsection{Routh Stability Criteria}

From characteristic Equation (9) we get

$$
\begin{gathered}
a_{0}=3261.5 \\
a_{1}=-2362 \\
a_{2}=11 \\
a_{3}=1 \\
b_{1}=a_{1}-\frac{a_{3} a_{0}}{a_{2}}=-5634.5
\end{gathered}
$$

Since, there is a negative element in the first column of Table 1. Therefore, System (5) is unstable.

\subsubsection{Hurwitz Stability Criteria}

We use determinants formed from coefficients of the characteristic Equation (9) we get:

$$
\begin{gathered}
\Delta_{1}=a_{2}=11>0 \\
\Delta_{2}=\left|\begin{array}{ll}
a_{2} & a_{0} \\
a_{3} & a_{1}
\end{array}\right|=-29243.5<0 \\
\Delta_{3}=\left|\begin{array}{ccc}
a_{2} & a_{0} & 0 \\
a_{3} & a_{1} & 0 \\
0 & a_{2} & a_{0}
\end{array}\right|=-95377675.25<0
\end{gathered}
$$

Since there is values of minors are less than zero, so the system (5) is unstable.

Table 1. Routh array of system (5).

\begin{tabular}{ccc}
\hline$\lambda^{3}$ & 1 & -2362 \\
\hline$\lambda^{2}$ & 11 & 3261.5 \\
$\lambda^{1}$ & -5634.5 & 0 \\
$\lambda^{0}$ & 3261.5 & 0 \\
\hline
\end{tabular}




\subsubsection{Lapiynuov Function}

Lapiynuov function and its derivatives for system (5) yield (10) \& (11).

We assume that

$$
\begin{gathered}
V\left(x_{1}, x_{2}, x_{3}\right)=\frac{1}{2}\left(x_{1}^{2}+x_{2}^{2}+x_{3}^{2}\right) \\
\dot{V}\left(x_{1}, x_{2}, x_{3}\right)=\frac{\partial v}{\partial x_{1}} \frac{\mathrm{d} x_{1}}{\mathrm{~d} t^{\prime}}+\frac{\partial v}{\partial x_{2}} \frac{\mathrm{d} x_{2}}{\mathrm{~d} t^{\prime}}+\frac{\partial v}{\partial x_{3}} \frac{\mathrm{d} x_{3}}{\mathrm{~d} t^{\prime}} \\
\dot{V}\left(x_{1}, x_{2}, x_{3}\right)=x_{1} \dot{x}_{1}+x_{2} \dot{x}_{2}+x_{3} \dot{x}_{3}
\end{gathered}
$$

By substituting (5) in Equation (11) we get:

$$
\dot{V}\left(x_{1}, x_{2}, x_{3}\right)=-10 x_{1}^{2}+306.5 x_{1} x_{2}-30 x_{1} x_{2} x_{3}-x_{3}^{2}
$$

Since $\dot{V}\left(x_{1}, x_{2}, x_{3}\right)>0$, therefore the system (5) is unstable.

\subsection{Lapiynuov Exponent and Lapiynuov Dimension}

The values of Lapiynuov exponents are:

$$
L_{1}=2.509426, L_{2}=0.132019, L_{3}=-11.818787
$$

Therefore, the Lapiynuov dimension "Kaplan-Yorke dimension" is:

$$
D_{L}=2+\frac{L_{1}+L_{2}}{\left|L_{3}\right|}=2.22349544
$$

So the system is hyperchaotic system, as shown in Figure 4.

\section{The Simulation Results}

In this section, the designed circuit to implement the chaotic system (1) was simulated by electronic simulation MultiSIM 10 program; Figure 5 shows sketch of the designed circuit of chaotic system (1)

The outputs voltages signals $V_{x_{1}}, V_{x_{2}}, V_{x_{3}}$ versus time, and phase portraits of the attractors are presented, in Figure 6 which shows the chaotic behaviors of the circuit.

By comparing Figure 6 with Figure 2 \& Figure 3 obtained by Matlab numerically, we conclude that between numerical simulation and experimental achievements there is a well qualitative agreement.

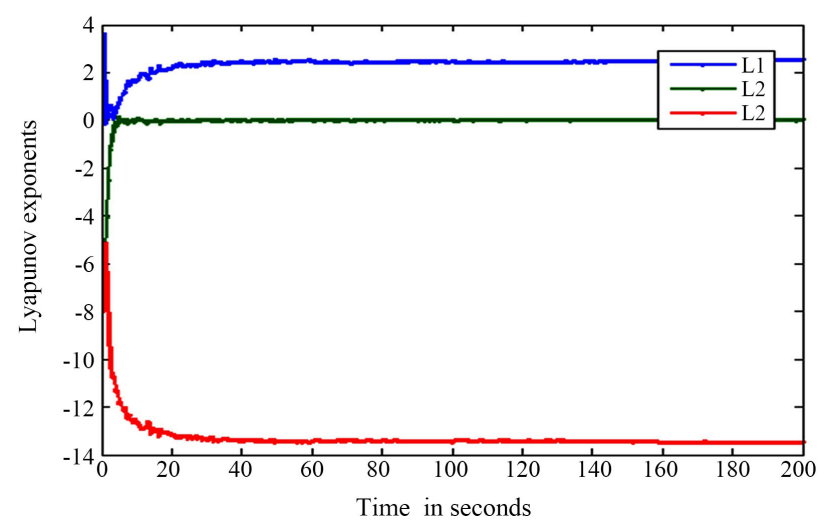

Figure 4. Lapiynuov exponent of system (5). 


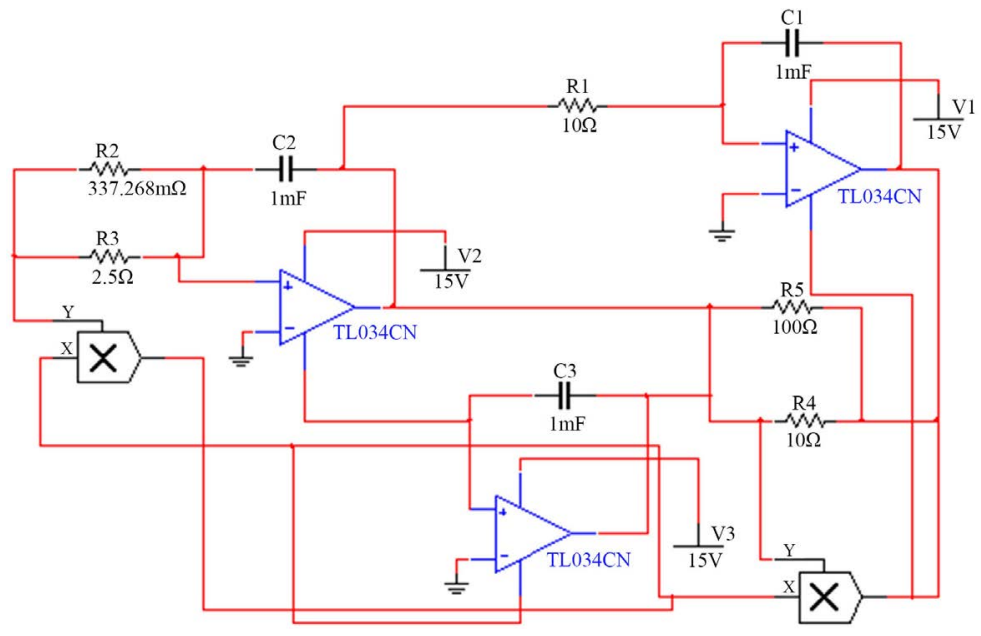

Figure 5. Sketch of the designed circuit of chaotic system (1).
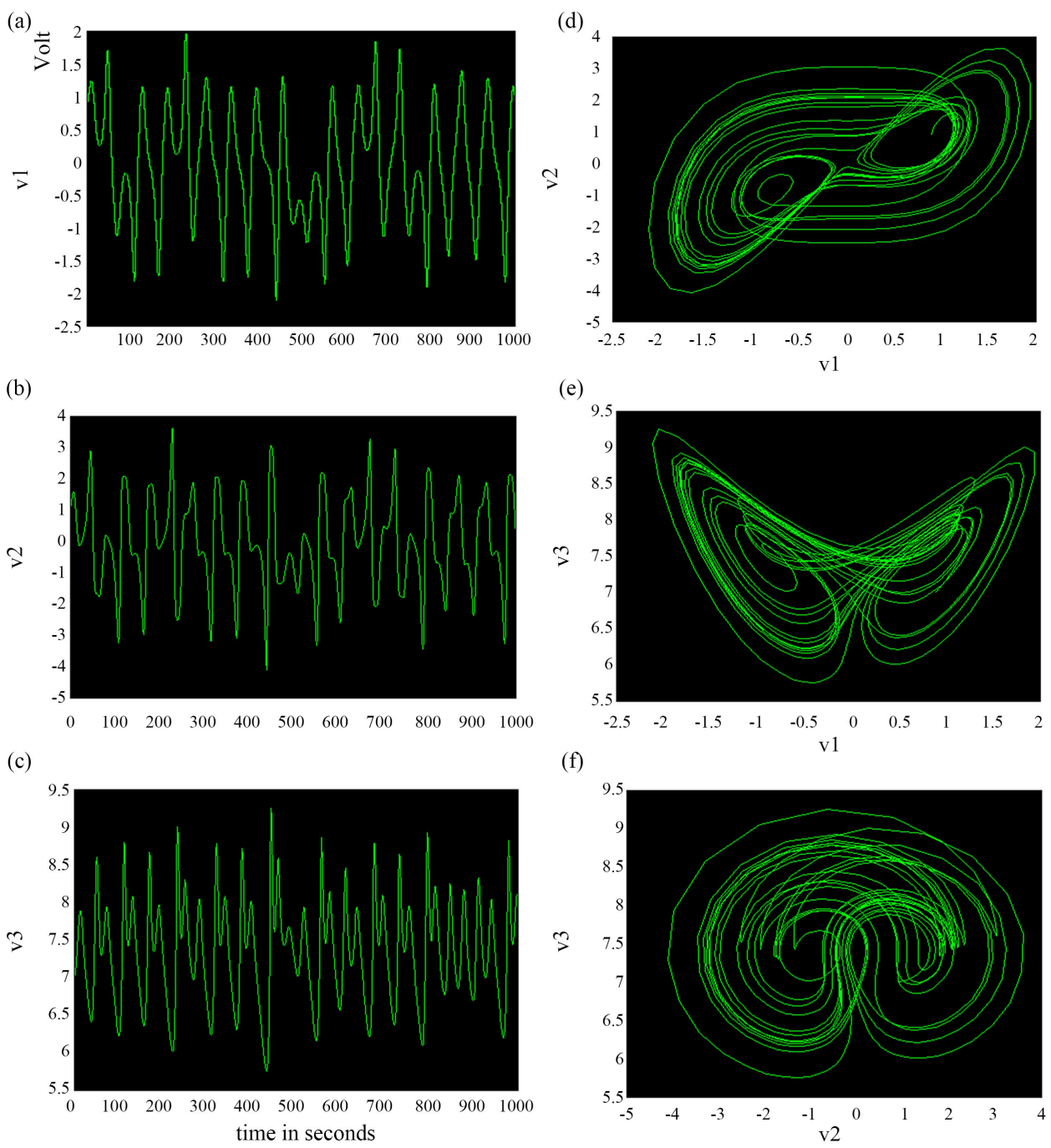

Figure 6. The left side shows the output voltage signals in (a) ( $V_{1}$ versus time), (b) ( $V_{2}$ versus time), (c) ( $V_{3}$ versus time); While right side shows the phase portraits of the designed electronic circuit in (d) $\left(V_{1}, V_{2}\right)$ plane, (e) $\left(V_{1}, V_{3}\right)$ plane, (f) $\left(V_{2}, V_{3}\right)$ plane. 


\section{Conclusion}

A three-dimensional chaotic system with two equilibrium points is analyzed. The Lyapunov dimension of the chaotic system is computed as $D_{L}=2.223495$, which shows that the system is hyperchaotic system. An electronic circuit is designed to implement chaotic system (1). Then, the basic characteristics of the proposed circuit model were analyzed by equilibrium points, stability analysis methods (such as characteristic equation roots, Routh criterion and Lapiynuov function); all these methods proved the instability of the new designed circuit (3), Lyapunov exponents and Kaplan-Yorke dimension that shows chaotisity of the designed circuit. The designed circuit is simulated by electronic simulation MultiSIM 10 program; the results show that there is a well qualitative agreement between the experimental achievements and numerical simulation which is obtained by using Matlab.

\section{Acknowledgements}

The authors are very grateful to the University of Mosul/College of Computer Sciences and Mathematics for their provided facilities, which helped to improve the quality of this work.

\section{Conflicts of Interest}

The authors declare no conflicts of interest regarding the publication of this paper.

\section{References}

[1] Aziz, M.M. and Hamid, M.A. (2019) The possibility of Increasing the Predictability Indices after Control of 3-D Continuous-Time system. International Conference on Computing and Information Science and Technology and Their Applications, Kirkuk, 3-5 March 2019, 1-5.

[2] Aziz, M.M. and AL-Azzawi, S.F. (2017) Hybrid Chaos Synchronization between Different Hyperchaotic Systems via Two Approaches. Optik, 138, 328-340. https://doi.org/10.1016/j.ijleo.2017.03.053

[3] Aziz, M.M. and Al-Nuaimi, Z.A. (2013) Stability and Hop-Bifurcation for Diabetes Model. International Journal of Electronics Communication and Computer Engineering, 4, 805-809.

[4] Aziz, M.M. and Faraj, M.N. (2012) Numerical and Chaotic Analysis of Chua's Circuit. Journal of Emerging Trends in Computing and Information Science, 3, 783-791.

[5] Aziz, M.M. (2018) Stability Analysis of Mathematical Model. International Journal of Science and Research (IJSR), 7, 147-148.

[6] Lamamra, K., Vaidyanathan, S., Putra, W.T., Darnila, E., Sambas, A. and Mujiarto (2020) A New 3-D Chaotic Jerk System with Four Nonlinear Terms, Its Backstepping Synchronization and Circuit Simulation. Journal of Physics: Conference Series, 1477, Article ID: 022017. https://doi.org/10.1088/1742-6596/1477/2/022017

[7] Salam, Md.A. and Rahman, Q.M. (2018) Fundamentals of Electrical Circuit Analysis. Springer Nature Singapore Private Limited, Singapore.

[8] Aziz, M.M. and Merie, D.M. (2020) Stability and Adaptive Control with Synchronization of 3-D Dynamical System. Open Access Library Journal, 7, 1-18. 